\title{
¿Estamos tan mal? La Educación Musical en España respecto a Alemania y
} Austria

Are we so bad? Music Education in Spain compared to Germany and Austria

\author{
Borja Mateu Luján \\ Borja.Mateu@uv.es \\ Departamento de Educación Comparada e Historia de la Educación \\ Universidad de Valencia \\ Valencia, España \\ ORCID: https://orcid.org/0000-0003-0961-9435
}

doi: 10.7203/LEEME.48.21552

Recibido: 17-09-00 Aceptado: 19-11-2021. Contacto y correspondencia: Borja Mateu-Luján, Departamento de Educación Comparada e Historia de la Educación, Universidad de Valencia, Avda. Blasco Ibañez, 30, C.P. 46010 Valencia, España.

\section{Resumen}

El presente artículo compara la educación musical que se oferta dentro de la educación obligatoria en España, Alemania (NordrheinWestfalen, NRW) y Austria. Para ello, se comparan las horas dedicadas a la educación musical en cada país, los contenidos que se imparten y la formación inicial de sus docentes. Los resultados muestran cómo la educación musical española, pese a que su enseñanza es optativa, tiene un horario admisible si lo comparamos con los de Alemania (NRW) y Austria. Del mismo modo, si nos centramos en los aprendizajes curriculares, también encontramos que son similares a los de Austria tanto para la Educación Primaria como para la Secundaria. Otro aspecto por destacar es que, respecto a la formación inicial, el profesorado español y alemán (NRW) de Educación Primaria es semiespecialista en Música, a diferencia del de Austria que es generalista. Por otro lado, en Alemania (NRW) y Austria el profesorado de Educación Secundaria es especialista en dos materias, mientras que en España lo es exclusivamente en Música. En consecuencia, y a raíz del estudio que se realiza en este artículo, parece que las diferencias musicales con respecto a estos dos países podrían deberse a otros aspectos como a la menor duración de la formación inicial del profesorado español, el nivel socioeconómico de las familias o el contexto cultural.

Palabras clave: Educación musical; educación comparada; educación obligatoria; currículo.

\begin{abstract}
This article compares the Music Education offered within the compulsory education in Spain, Germany (Nordrhein-Westfalen, NRW), and Austria. To do this, the hours devoted to Music Education in each country, the content taught, and the initial training of their teachers are compared. The results show how Spanish Music Education, even though its teaching is optional, has an acceptable schedule if we compare it with those of Germany (NRW) and Austria. Similarly, if we focus on curricula learnings, we also find that they are similar to those in Austria for both primary and secondary education. Another aspect worth noting is with regard to initial training; while Spanish and German (NRW) primary school teachers are semi-specialists in music, in Austria they are generalists. As for secondary education, while in Germany (NRW) and Austria teachers are specialists in two subjects, in Spain they are exclusively specialists in music. Consequently, as a result of this article, it seems that the musical differences with respect to these two countries could be due to other aspects such as the shorter duration of the initial training of Spanish teachers, the socioeconomic level of the families, or the cultural context.
\end{abstract}

Key words: Music Education; Comparative Education, Compulsory Education; Curriculum 


\section{Introducción}

En los últimos años, los estudios internacionales de educación vienen determinando que las asignaturas de Arte y Humanidades están perdiendo presencia dentro de los sistemas educativos nacionales (Ruiz y García-Álvarez, 2016). En este sentido, la educación musical se está viendo muy afectada, tal y como viene observando Aróstegui $(2016 ; 2020)$. Este declive proviene de organismos internacionales como la OCDE en razón de las políticas educativas que aplica. Así, la OCDE, a través de las pruebas PISA, está orientando la opinión pública y la de los políticos hacia las asignaturas STEM y los idiomas provocando que se ignore la importancia y la necesidad del resto de materias (Chrystosomou, 2015).

En contraposición con estas tendencias educativas que desdibujan la importancia de las Artes y Humanidades dentro de los sistemas educativos, encontramos otras políticas que sí se la otorgan como las impulsadas desde la UNESCO a través de los Objetivos de Desarrollo Sostenible. En este sentido, el objetivo 4 de esta Agenda 2030 es el de «Garantizar una educación inclusiva y equitativa de calidad y promover oportunidades de aprendizaje permanente para todos» (UNESCO, 2015, p.33). Obviamente, desde este planteamiento educativo la educación musical se convierte en una materia imprescindible, puesto que la inclusión, el respeto a la diversidad, la tolerancia, la empatía, la colaboración y la comunicación son cualidades que se han visto desarrolladas a partir de los programas artísticos (Brasche y Thorn, 2018; Campbell, 2020). Asimismo, la educación musical puede contribuir a desarrollar el sentido estético y cívico, la capacidad de construir opiniones y criterios, y fomentar el pensamiento crítico y emancipador (Bate, 2020; Fernández-Jiménez y Jorquera-Jaramillo, 2017). Desde este punto de vista educativo, para que la educación cumpla su función de justicia social, la educación musical debería estar presente en la formación obligatoria de los más jóvenes con independencia de su situación geográfica, el nivel socioeconómico familiar, el género o la raza (Horsley, 2015). Para ello, la formación inicial docente también debería incrementar los programas que fomenten la diversidad cultural (Escalante, 2020; Palmer, 2018; Salvador y Kelly-McHale, 2017).

En el contexto europeo, se han encontrado evidencias de que respecto a la educación musical se combinan ambos posicionamientos. Por un lado, encontramos que uno de los principales objetivos de la Unión Europea en materia de educación -en línea con la OCDE- es el de aumentar la empleabilidad a través de competencias transversales como la iniciativa emprendedora, las aptitudes digitales o los idiomas (Comisión Europea, 2012). Si bien, por otro lado, López-García y De Moya-Martínez (2017) han constatado que la Unión Europea también presta una especial importancia a la educación musical a través de sus diferentes organismos. Como resultado de este doble posicionamiento respecto a la educación musical los estados miembros han generado una gran disparidad entre ellos (López-García y De Moya-Martínez, 2017).

\footnotetext{
@ Borja Mateu Luján. The content of this article is the sole responsibility of the authors. The Revista Electrónica de LEEME and Universitat de València are not liable for any legal actions that may arise involving the article's content. Revista Electrónica de LEEME - Lista Electrónica Europea de Música en la Educaciónhttp://ojs.uv.es/index/php/LEEME/index ISSN: 1575-9563. Editores: Universidad de Valencia y Jesús Tejada. Visibilidad de esta revista: SCOPUS, Emerging Sources Citation Index (Clarivate), EBSCO, CINDOC (CSIC), Citefactor, COPAC, Dialnet, DICE (CSIC), DOAJ, e-revistas (CSIC), EBSCO Premier, ERIH+, Gale Cengage Learning, IN-RECS, IRESIE, LATINDEX, MIAR, OCLC Worldcat, RESH, REDIB, RILM Core Journals, SUDOC, ULRICHS. Esta revista es de acceso libre mediante licencia Creative Commons 4.0 CC by. Política de archivado: etiqueta verde SHERPA-ROMEO.
} 
Para comprobar si existe tal disparidad en el tratamiento de la educación musical entre los diferentes estados miembros de la Unión Europea, el presente artículo tiene el objetivo de realizar un estudio comparado entre la educación musical que se imparte en España, Alemania y Austria dentro de la educación obligatoria. Empero, la educación comparada no tiene el objetivo de homogeneizar sistemas educativos, sino conseguir extrapolar aquellos elementos que puedan ayudar a resolver problemas o mejorar situaciones de los diferentes territorios analizados (Arnove et al., 2016).

La hipótesis inicial es que estos países con una gran cultura musical presentarán, con respecto a España, una mayor atención a la educación musical dentro de sus respectivos sistemas educativos, así como a la formación inicial de sus docentes de Música. No en vano cabe recordar que en el contexto español, durante los cambios legislativos acontecidos en las últimas décadas, la educación musical ha ido perdiendo presencia y estatus ofreciéndose como una asignatura optativa a merced de los distintos currículos autonómicos (Morales, 2017).

\section{Método}

El método comparado aplicado a la educación musical se ha convertido en una metodología indispensable para conocer, aportar y analizar las estrategias puestas en práctica tanto en el ámbito nacional como en el internacional con el fin de establecer una correcta toma de decisiones que ayude a mejorar la educación musical dentro del sistema obligatorio de enseñanza (Esteve-Faubel, 2019). Con este propósito, en los últimos años se han publicado numerosas investigaciones que realizan comparaciones entre países sobre los currículos de música (Garvis et al., 2017; Iotova y Siebenaler, 2018; Mateu-Luján, 2021a; Vicente-Nicolás y Kirihara, 2012) o sobre la formación inicial de sus docentes (Carmona Fernández y Jurado Fernández, 2010; Domínguez-Lloria y Pino-Juste, 2020; Herrera Torres et al., 2010; Rodríguez-Quiles, 2010).

Tomando como referencia los trabajos anteriores y para alcanzar los objetivos determinados, en el presente artículo se ha desarrollado un estudio comparado entre los territorios de España, Alemania y Austria. No obstante, cabe matizar que debido a que Alemania se trata de un Estado federal, únicamente se ha analizado el estado de Nordrhein-Westfalen (NRW). En el caso de España, aunque es cierto que también se trata de un país muy descentralizado, existen estudios recientes nacionales sobre la educación musical que nos permiten comparar el país en su conjunto (se destacan los de Casanova-López y Serrano-Pastor, 2018; López-García, 2018 y Mateu-Luján, 2021b). Por último, en Austria, pese a disponer de una organización federal, su legislación educativa es nacional.

En concreto, se analiza el número de horas semanales en las que se imparte la educación musical dentro de la educación obligatoria de cada territorio, los cursos en los cuales se ubican dentro del horario de Educación Primaria y Secundaria, la formación inicial del profesorado y la 
estructuración de los contenidos. La información recopilada se ha extraído a partir de las legislaciones educativas vigentes en cada uno de los territorios, la literatura científica disponible y las consultas a expertos o entidades de cada país. Siguiendo las principales directrices del método comparado (Phillips y Schweisfurth, 2014), el presente estudio se estructura en tres fases correspondientes con los apartados 3., 4. y 5. En la primera fase, se recopila y analiza la información, en la segunda se realiza la yuxtaposición de los datos analizados y, finalmente, en la última fase se comparan y discuten los resultados obtenidos.

\section{La educación Musical en los distintos contextos}

\subsection{El contexto español}

El sistema educativo español dispone de un marco legislativo general (LOMCE) (Jefatura del Estado, 2013) que se concreta en cada una de las comunidades autónomas, aunque su margen de actuación está bastante acotado. La organización del sistema educativo español se considera semicomprensiva, puesto que solo en los últimos años de la Educación Secundaria Obligatoria (ESO) se introducen ciertos itinerarios educativos que permiten al alumnado elegir de acuerdo con sus motivaciones o su rendimiento académico. Por lo que a la organización de las etapas se refiere, cabe destacar que en España encontramos dos: la Educación Primaria y la ESO. La primera de ellas tiene una duración de 6 cursos y abarca las edades comprendidas entre los 6 y los 12 años. La segunda tiene una duración de cuatro cursos y aborda las edades de 12 a 16 años.

Respecto a la educación musical, cabe señalar que la normativa estatal propone esta materia como optativa tanto para la Educación Primaria como para la ESO. Así, de acuerdo con esta legislación, podría ocurrir que un ciudadano español terminara sus estudios obligatorios sin haber recibido ni tan solo una clase de educación musical (Carrillo Aguilera et al., 2017). Por suerte, existen estudios científicos que corroboran que la desaparición de la educación musical durante la LOMCE (2013) no ha sido tan catastrófica como cabría esperar (Belletich et al., 2016).

En Educación Primaria, cabe recordar que la educación musical debe compartir sus horas asignadas con la Educación Plástica, ya que ambas materias conforman la asignatura de Educación Artística (Real Decreto 126/2014). En este sentido, Casanova-López y Serrano-Pastor (2018) y López-García (2018) determinan que la mayoría de los centros españoles dedican dos sesiones de Educación Artística a la semana, lo que presupone que una de ellas sería para la educación musical y la otra para la Educación Plástica. Según estos autores, la duración de las sesiones de música semanales oscilan entre $45 \mathrm{~min}$ y una hora, aunque existen casos particulares con menor dedicación como la Comunidad de Madrid y la Región de Murcia o con mayor dedicación como Canarias, Castilla y León, Cataluña, La Rioja o la Comunidad Valenciana. 
En la Educación Secundaria Obligatoria, la organización de la educación musical presenta algunas diferencias respecto a Educación Primaria. En primer lugar, la educación musical es considerada como una asignatura en sí misma y no está englobada dentro de la Educación Artística (Real Decreto 1105/2014). Y, en segundo lugar, el número de sesiones que se imparte por semana tiene una gran variedad entre comunidades autónomas. No obstante, se ha determinado que en España se cursa de forma obligatoria aproximadamente con una media de 3 horas y 9 minutos durante el primer ciclo de la ESO equivalentes a cuatro sesiones semanales para toda la etapa (Mateu-Luján, 2021b). En este sentido, Aragón, Cantabria y la Comunidad Valenciana son las que más tiempo dedican de forma obligatoria, mientras que las Islas Canarias es la que menos (sin contar Ceuta y Melilla) (Mateu-Luján, 2021b).

La distribución de los aprendizajes se organiza por bloques de contenido y para cada uno de estos bloques la legislación estatal únicamente prescribe criterios de evaluación y estándares de aprendizaje evaluables. No obstante, los diferentes territorios autonómicos, dentro de los márgenes establecidos, suelen concretar algunos elementos como los contenidos o las orientaciones metodológicas. En concreto, para Educación Primaria los bloques establecidos son: 1. Escucha, 2. La interpretación musical, y 3. La música, el movimiento y la danza (Real Decreto 126/2014). En la Educación Secundaria, la legislación determina 4 bloques de contenido, a saber: 1. Interpretación y creación, 2. Escucha, 3. Contextos musicales y culturales, y 4. Música y tecnologías (Real Decreto 1105/2014).

Respecto a la formación inicial docente en España, encontramos dos modelos distintos atendiendo a la etapa en la que impartirán su docencia (Morales, 2017). En Educación Primaria, el futuro profesorado recibe una formación de 240 ECTS de los cuales solo 30 ECTS se dedican a la educación musical. Respecto a la Educación Secundaria, el futuro profesorado debe cursar estudios universitarios o equivalentes relacionados con la música y, una vez finalizados, cursar un Máster de Profesorado en Educación Secundaria de 60 ECTS.

\subsection{El contexto alemán (NRW)}

Realizar un análisis de la educación musical en Alemania se trata de una tarea muy compleja, puesto que, al ser una república federal, en cada uno de los estados el sistema educativo se rige por su propia legislación. En consecuencia, tal y como se anunciaba en la metodología, en el presente artículo únicamente se analiza la educación musical dentro del sistema educativo de Nordrhein-Westfalen (NRW). Sin duda, cualquier estado podría haberse utilizado en este artículo debido a la gran cultura musical existente en cada uno de ellos. No obstante, en aras de conseguir 
una mayor representatividad se ha elegido el territorio de NRW por ser el más poblado de Alemania ${ }^{1}$.

Las características principales que definen la educación en Alemania, en general y, por ende, dentro de Nordrhein-Westfalen, son que tienen una Educación Primaria de corta duración (4 años) y que la Educación Secundaria incorpora numerosos itinerarios educativos (Eurydice, 2020). Estos itinerarios dividen el alumnado en función de sus aptitudes y de sus motivaciones. Así, el sistema educativo alemán se trata de un sistema diferenciado. De este modo, se añade una nueva variable al estudio de la educación musical en Alemania (NRW), puesto que en cada una de estas escuelas de Educación Secundaria el horario de la educación musical puede variar. La extensión de los estudios de Educación Secundaria abarca desde los 10 a los 19 años. Esta se divide en la Sekundarstufe I (10-16 años) y la Sekundarstufe II (16-19 años). Respecto a la Sekundarstufe II, cabe destacar que no es objeto de este estudio, puesto que, aunque es obligatoria en NRW, es equivalente al Bachillerato español.

En lo referente a la educación musical, en la etapa Primaria la distribución horaria se establece de forma muy clara según el Ministerium für Schule und Bildung des Landes NordrheinWestfalen (2005). En cada uno de los dos primeros cursos, el alumnado deberá cursar entre 3 y 4 sesiones semanales de 45 minutos a dividir entre Música y Plástica. Por los que respecta a los cursos de tercero y cuarto de Educación Primaria, cabe destacar que para cada uno de ellos se establecen 4 sesiones semanales de 45 minutos a repartir entre Música y Plástica.

En la etapa Secundaria, la educación musical es muy distinta debido a su organización diferenciada del sistema educativo. Por un lado, la Música está insertada dentro de la Educación Artística y, por el otro, la asignatura de Educación Artística está compuesta por diversas materias en función del itinerario en el que se imparta. En la Tabla 1, se presenta el número de sesiones semanales en los que se ofrece la materia de Educación Artística para cada uno de los itinerarios de la Sekundarstufe I.

El currículo de educación musical tanto de Educación Primaria como de Educación Secundaria en el territorio de Nordrhein-Westafalen contiene una justificación, objetivos, contenidos, competencias y criterios de evaluación (Ministerium für Schule und Bildung des Landes Nordrhein-Westfalen, 2008; 2011; 2012b; 2012c; 2012d; 2019). Por lo que respecta a la Educación Primaria, los contenidos y las competencias se prescriben para dos momentos, el final del segundo curso y el final de la etapa. Estos se articulan en tres grandes bloques: Musik hören (escuchar música), Musik manchen (hacer música) y Musik umsetzen (poner la música en práctica) (Ministerium für Schule und Bildung des Landes Nordrhein-Westfalen, 2008).

\footnotetext{
${ }^{1}$ Fuente: https://www.statista.com/statistics/1127686/population-by-federal-state-germany/
}

@ Borja Mateu Luján. The content of this article is the sole responsibility of the authors. The Revista Electrónica de LEEME and Universitat de València are not liable for any legal actions that may arise involving the article's content. Revista Electrónica de LEEME - Lista Electrónica Europea de Música en la Educación-. http://ojs.uv.es/index/php/LEEME/index ISSN: 1575-9563. Editores: Universidad de Valencia y Jesús Tejada. Visibilidad de esta revista: SCOPUS, Emerging Sources Citation Index (Clarivate), EBSCO, CINDOC (CSIC), Citefactor, COPAC, Dialnet, DICE (CSIC), DOAJ, e-revistas (CSIC), EBSCO Premier, ERIH+, Gale Cengage Learning, IN-RECS, IRESIE, LATINDEX, MIAR, OCLC Worldcat, RESH, REDIB, RILM Core Journals, SUDOC, ULRICHS. Esta revista es de acceso libre mediante licencia Creative Commons $4.0 \mathrm{CC}$ by. Política de archivado: etiqueta verde SHERPA-ROMEO. 
Tabla 1. Sesiones semanales de Educación Artística en la Sekundarstufe I de Alemania (NRW)

\begin{tabular}{|c|c|c|c|}
\hline Escuela Secundaria & Materias & $\begin{array}{c}10-12 \text { años } \\
\text { Orientierungsstufe }\end{array}$ & $12-16$ años \\
\hline Hauptschule & $\begin{array}{l}\text { Arte, música y } \\
\text { diseño textil }\end{array}$ & 8 & 8 \\
\hline Gesamtschule & Arte y música & 8 & 8 \\
\hline Realschule & $\begin{array}{l}\text { Arte, música y } \\
\text { diseño textil }\end{array}$ & 8 & 8 \\
\hline Gymnasium (G8)* & Arte y música & 8 & 6 \\
\hline Gymnasium (G9) & Arte y música & 7 & 10 \\
\hline
\end{tabular}

Fuente: Ministerium für Schule und Bildung des Landes Nordrhein-Westfalen (2012a)

*Este itinerario académico termina a los 15 años y se extinguirá en el curso 2021/22

En la etapa Secundaria, la organización de estos bloques es diferente. Para cada uno de los itinerarios, la educación musical se organiza en tres áreas de contenido: Bedeutungen von Musik (significados de la música), Entwicklungen von Musik (desarrollos musicales) y Verwendungen von Musik (usos de la música) (Ministerium für Schule und Bildung des Landes Nordrhein-Westfalen, 2011; 2012b; 2012c; 2012d; 2019). Dentro de cada una de estas áreas de contenido, se articulan competencias de producción, recepción y reflexión. Si comparamos los currículos de educación musical en cada uno de los itinerarios de la Sekundarstufe I, se observa que no existe una gran diferencia entre aprendizajes. No obstante, dependiendo del itinerario, sí que tendrán una mayor o menor profundidad de acuerdo con el horario establecido y si ese tipo de escuela incorpora conocimientos del diseño textil dentro del área de Educación Artística.

La formación inicial del profesorado de Alemania es una de las más completas de Europa tanto para el profesorado de Primaria como para el de Secundaria. Además, ambas tienen una duración similar. Este es un elemento muy interesante, ya que en la mayoría de los países la formación inicial del profesorado de Secundaria tiene una formación más completa que el de Primaria. El futuro profesorado debe cursar un grado universitario de 3 años de duración, después un Máster de educación de dos años y, finalmente, debe realizar prácticas escolares remuneradas (Referendariat) durante 18 meses (Lehmann-Wersmser, 2015). En referencia a la capacitación docente para la educación musical, cabe destacar que el profesorado de Educación Primaria puede formarse como especialista en Música, pero antes debe haber sido formado en Lengua y Matemáticas. En referencia al profesorado de Educación Secundaria, puede ser especialista en Música, pero siempre deberá elegir otra materia como segunda opción (Rodríguez-Quiles, 2010). Del mismo modo, podría elegir otra materia como primera opción y Música como segunda. La 
diferencia entre elegir Música como primera opción o segunda recae en el número de horas de formación que recibe para cada una de las asignaturas ${ }^{2}$.

\subsection{El contexto austríaco}

Austria tiene unas grandes similitudes con Alemania. La primera de ellas obviamente es que ambos países comparten la cultura germana. Pero, también existe otro elemento interesante y es que ambos países son una república federal. No obstante, cabe destacar que en materia educativa Austria dispone de una sola ley educativa que articula todo el territorio, la Bundesgesetzblatt für die Republik Österreich (2017). Respecto a la educación obligatoria en Austria, esta comprende desde los 5 a los 15 años (Eurydice, 2020). Si bien, el primer curso de educación obligatoria se corresponde con el último de la etapa Infantil, por lo que no ha sido considerado para este estudio - asimismo tampoco depende del Ministerio de Educación-. En concreto, la Educación Primaria ocupa 4 cursos y abarca entre los 6 y los 10 años. Por último, la Educación Secundaria se compone de 6 cursos (aunque el último no es obligatorio) y tiene una estructura diferenciada. Para los 4 primeros cursos de esta etapa Secundaria, existen dos itinerarios el Allgemeinbildende Höhere Schule (académico) y la Neue Mittelschule (integra conocimientos académicos y profesionales); mientras que, para los dos últimos cursos de esta etapa Secundaria, existen 2 itinerarios académicos y 3 vocacionales (Eurydice, 2020).

En relación con el horario propuesto para la educación musical, cabe destacar que dentro de la Educación Primaria en Austria se oferta una sesión semanal de 50 minutos en cada uno de los cuatro cursos (Bundesministerin für Unterricht, 2012a). En la Educación Secundaria, existe una mayor variación horaria entre cada uno de los cursos (Bundesministerin für Bildung, 2004; Bundesministerin für Unterricht, 2012b). Así, durante los dos primeros cursos, el alumnado austriaco recibe 2 sesiones semanales de 50 minutos de educación musical. En los dos siguientes, el horario de Música se reduce a la mitad, puesto que en este momento el alumnado recibe 1 sesión semanal de 50 minutos. Por último, en los quinto y sexto cursos de la Educación Secundaria, el alumnado puede cursar 3 sesiones de 50 minutos de educación musical de forma optativa dependiendo del itinerario (Bundesministerin für Bildung, 2004), aunque obviamente el sexto curso ya no forma parte de la educación obligatoria.

Todos los currículos educativos citados en el párrafo anterior mantienen la misma estructura con independencia del nivel al que se refieran. Estos contienen una justificación, contenidos, material didáctico y principios didácticos. Respecto a la distribución de los contenidos encontramos que es similar para los dos ciclos que componen la Educación Primaria, estos son: Singen (cantar), Musizieren (hacer música), Hören (escuchar) y Bewegen zu Musik (moverse con

\footnotetext{
${ }^{2}$ Información proporcionada por el Ministerium für Schule und Bildung des Landes Nordrhein-Westfalen a través del correo Poststelle@msb.nrw.de

@Borja Mateu Luján. The content of this article is the sole responsibility of the authors. The Revista Electrónica de LEEME and Universitat de València are not liable for any legal actions that may arise involving the article's content. Revista Electrónica de LEEME - Lista Electrónica Europea de Música en la Educación-. http://ojs.uv.es/index/php/LEEME/index ISSN: 1575-9563. Editores: Universidad de Valencia y Jesús Tejada. Visibilidad de esta revista: SCOPUS, Emerging Sources Citation Index (Clarivate), EBSCO, CINDOC (CSIC), Citefactor, COPAC, Dialnet, DICE (CSIC), DOAJ, e-revistas (CSIC), EBSCO Premier, ERIH+, Gale Cengage Learning, IN-RECS, IRESIE, LATINDEX, MIAR, OCLC Worldcat, RESH, REDIB, RILM Core Journals, SUDOC, ULRICHS. Esta revista es de acceso libre mediante licencia Creative Commons 4.0 CC by. Política de archivado: etiqueta verde SHERPA-ROMEO.
} 
la música). Por lo que respecta a la distribución de contenidos en la Educación Secundaria, esta es mucho más amplia, a saber: Vokales Musizieren (creación música vocal), Instrumentales Musizieren (creación música instrumental), Bewegen (movimiento), Gestalten (crear), Hören (escuchar), Grundwissen (conocimientos básicos), Erweiterungsbereich (área de extensión, solo segundo ciclo). Por último, el alumnado que decida cursar música en los cursos de quinto y sexto recibirá contenidos de: Musikpraxis (práctica musical), Musikkunde (musicología) y Musikrezeption (recepción de la música).

Finalmente, encontramos que la formación inicial docente es diferente atendiendo al nivel educativo en el cual se imparta educación, ya que mientras para Educación Primaria el profesorado es generalista, para la Educación Secundaria es especialista (en 2 materias) (Domínguez-Lloria y Pino-Juste, 2020). Así, en Educación Primaria, el profesorado debe cursar un grado universitario de cuatro años junto con un Máster de un curso de duración. No obstante, este actualmente solo se considera obligatorio para poder acceder a contratos indefinidos. Respecto a la formación inicial del profesorado de Educación Secundaria, estos deben cursar un grado universitario de 4 años y después un Máster de 2 años de educación, que al igual que para Educación Primaria actualmente no es obligatorio, pero sí necesario para acceder a contratos indefinidos. Si bien, la previsión es que sea obligatorio en el $2029^{3}$.

\section{Yuxtaposición}

A continuación, se presenta la yuxtaposición de la información analizada en el apartado anterior. Si nos fijamos en la configuración de los tres sistemas educativos podemos observar cómo España tiene un sistema semicomprensivo frente a Alemania y Austria que tienen un modelo diferenciado. Como se ha comentado anteriormente, este hecho es muy relevante para el presente estudio, puesto que la educación musical que reciba el alumnado puede variar significativamente en función del itinerario educativo en el cual cursen sus estudios. Teniendo en cuenta el presente aspecto, en la Figura 1 se muestra el número mínimo (parte izquierda) y máximo (parte derecha) de sesiones de educación musical que se pueden cursar en cada etapa educativa para cada uno de los territorios.

A partir de los datos recopilados en la Figura 1, a continuación, se matizan aquellos aspectos más relevantes. En primer lugar, cabe destacar que en el gráfico solo se han contabilizado los cursos de educación obligatoria que oscilan entre los 6 y los 16 años. En este sentido, tal y como se ha comentado anteriormente, no se ha incorporado el primer curso de la educación infantil ni tampoco los tres últimos años de la educación obligatoria alemana dirigida al alumnado de entre 16 y 19 años.

\footnotetext{
${ }^{3}$ Información proporcionada por Helmut Schaumberger, coordinador de Austria en la EAS.

@ Borja Mateu Luján. The content of this article is the sole responsibility of the authors. The Revista Electrónica de LEEME and Universitat de València are not liable for any legal actions that may arise involving the article's content. Revista Electrónica de LEEME - Lista Electrónica Europea de Música en la Educación-. http://ojs.uv.es/index/php/LEEME/index ISSN: 1575-9563. Editores: Universidad de Valencia y Jesús Tejada. Visibilidad de esta revista: SCOPUS, Emerging Sources Citation Index (Clarivate), EBSCO, CINDOC (CSIC), Citefactor, COPAC, Dialnet, DICE (CSIC), DOAJ, e-revistas (CSIC), EBSCO Premier, ERIH+, Gale Cengage Learning, IN-RECS, IRESIE, LATINDEX, MIAR, OCLC Worldcat, RESH, REDIB, RILM Core Journals, SUDOC, ULRICHS. Esta revista es de acceso libre mediante licencia Creative Commons 4.0 CC by. Política de archivado: etiqueta verde SHERPA-ROMEO.
} 

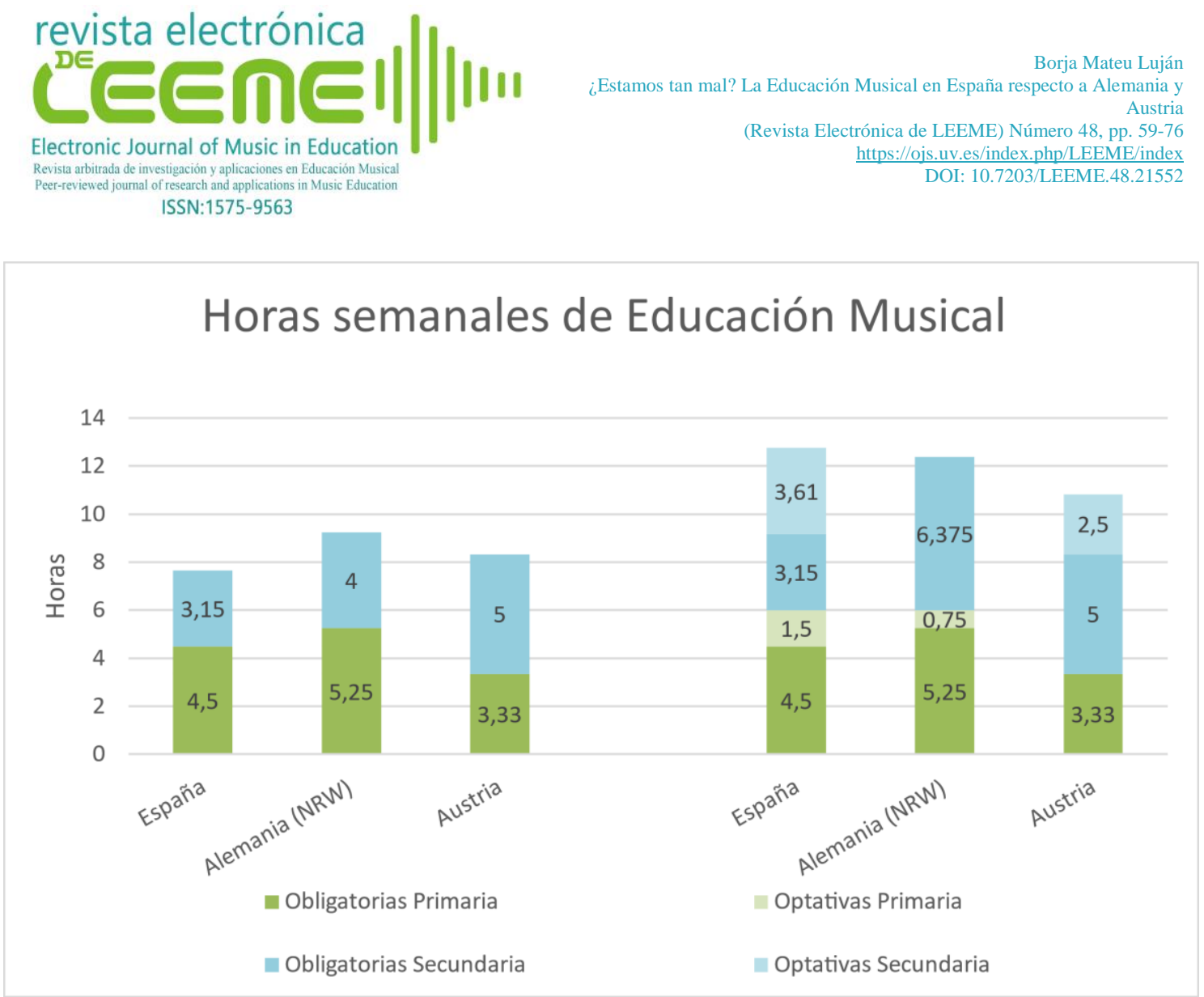

Figura 1. Horas semanales de educación musical para Educación Primaria y Secundaria en España, Alemania (NRW) y Austria

Debido a que los años de duración de la etapa Primaria y Secundaria son tan diferentes entre España y Alemania (NRW) y Austria, se ha establecido la comparación entre los sistemas educativos completos. Si nos fijamos en la parte izquierda del gráfico se observa cómo el territorio donde se cursan más horas de educación musical de forma obligatoria es Alemania (NRW) seguido de Austria y España. Obviamente, no es sorprendente que los países germanófonos tengan una mayor presencia de la educación musical, así la media española dista de 1 hora y 36 minutos respecto a la de Alemania (NRW) y de 40,8 minutos respecto a la de Austria (NRW).

Una vez analizada la parte izquierda del gráfico, nos centraremos ahora en la parte derecha. En este sentido, es interesante observar que al incorporar al gráfico el número de horas optativas se produzca una inversión total de los resultados respecto a la situación anterior. Así, podemos comprobar cómo en España el alumnado, dependiendo del lugar donde resida y sus motivaciones, podría estudiar las mismas horas de educación musical, o incluso más, que las que se imparten en Alemania (NRW) o Austria.

Aunque para el análisis del horario semanal dedicado a la educación musical no se ha realizado distinción entre etapas, en esta ocasión sí es conveniente hacerlo para el análisis de la distribución de los contenidos. Así, respecto al análisis de contenidos en Educación Primaria se puede observar que Austria y España mantienen una estructura similar. En este sentido, ambos

$@$ @orja Mateu Luján. The content of this article is the sole responsibility of the authors. The Revista Electrónica de LEEME and Universitat de València are not liable for any legal actions that may arise involving the article's content. Revista Electrónica de LEEME - Lista Electrónica Europea de Música en la Educación-. http://ojs.uv.es/index/php/LEEME/index ISSN: 1575-9563. Editores: Universidad de Valencia y Jesús Tejada. Visibilidad de esta revista: SCOPUS, Emerging Sources Citation Index (Clarivate), EBSCO, CINDOC (CSIC), Citefactor, COPAC, Dialnet, DICE (CSIC), DOAJ, e-revistas (CSIC), EBSCO Premier, ERIH+, Gale Cengage Learning, IN-RECS, IRESIE, LATINDEX, MIAR, OCLC Worldcat, RESH, REDIB, RILM Core Journals, SUDOC, ULRICHS. Esta revista es de acceso libre mediante licencia Creative Commons $4.0 \mathrm{CC}$ by. Política de archivado: etiqueta verde SHERPA-ROMEO. 
países construyen sus currículos con contenidos de audición musical, interpretación musical, y danza y movimiento. Respecto al currículo alemán (NRW) cabe señalar que entre sus bloques no incluye la danza, pero sí incorpora contenidos de análisis musical desde el punto de vista estético y formal. No obstante, aunque no exista un bloque específico de danza, sí se incluyen contenidos de esta índole distribuidos entre los distintos bloques.

En relación con la distribución de contenidos para Educación Secundaria, debe señalarse que España y Austria continúan tendiendo una distribución similar de los contenidos. Destacando únicamente dos cuestiones: España dedica un apartado exclusivo a la música y la tecnología, mientras que Austria lo dedica a la danza y el movimiento. Si bien, si realizamos un análisis más profundo más allá de la nomenclatura de los bloques, se observa cómo en la práctica los currículos de España y Austria presentan una gran similitud y, además, mantienen un equilibrio entre los diferentes contenidos de la educación musical. En contraposición, el currículo de Alemania (NRW) prioriza los contenidos relacionados con la práctica musical.

Para concluir solo queda realizar la yuxtaposición de la formación inicial docente de cada uno de los países. Así, mientras que todos los territorios analizados consideran que sus docentes de Educación Secundaria deben ser especialistas en educación musical -aunque en los casos de Alemania (NRW) y Austria deben serlo en dos materias-, para Educación Primaria existen tres modelos. El primero de ellos es el de Austria el cual utiliza docentes generalistas para impartir la educación musical, el segundo es el de España en el que el profesorado de Música recibe una formación semiespecialista, y, por último, encontramos el caso de Alemania (NRW) en el que el profesorado, además de ser capaz de impartir las asignaturas de Lengua y Matemáticas, puede escoger especializarse en docencia musical. En relación con la duración de cada formación inicial, cabe destacar que Alemania (NRW) es la más larga tanto para Educación Primaria como Secundaria. Ambos docentes disponen de una formación inicial de 3 cursos de Grado, 2 de Máster y 18 meses de prácticas escolares remuneradas. A esta formación inicial, le sigue la de Austria la cual abarca 4 cursos de Grado y un curso de Máster (contratos indefinidos) para Educación Primaria y 4 cursos de Grado y dos de Máster (contratos indefinidos, obligatorio en 2029) para Educación Secundaria. Finalmente, encontramos el caso español en el que la formación inicial del profesorado de Educación Primaria consta de 4 cursos de Grado, y la formación Secundaria de 4 cursos de Grado más un curso de Máster.

\section{Discusión y conclusiones}

El presente artículo partía de la hipótesis de que la educación musical recibía una menor atención dentro del sistema educativo español que en países como Alemania (NRW) o Austria. Si bien, esta hipótesis se cumple a nivel general, es interesante analizar los elementos comparados por separado. Así, encontramos que en España se imparte aproximadamente una sesión semanal menos de música que en Austria (40,8 minutos) y dos menos que en Alemania (NRW) (1 hora y 
36 minutos) durante la educación obligatoria. No obstante, tal y como se ha demostrado en este artículo, podría ocurrir que un alumno español, dependiendo del territorio donde viva y las optativas que curse, reciba más horas de educación musical que el alumnado de Alemania (NRW) o Austria.

El siguiente aspecto destacable es que existe una gran similitud en relación con los contenidos musicales que se imparten en Austria y España. En este sentido, mientras España y Austria persiguen un equilibrio entre los diferentes aprendizajes de la educación musical, en Alemania (NRW) se prioriza el análisis estético y formal de la música en Educación Primaria y la práctica musical en Secundaria. Empero, si existe un hecho diferenciador entre España y el resto de los países, este hace referencia a que la formación inicial del profesorado español tenga una duración más corta que la que se imparte en Alemania (NRW) y en Austria (DomínguezLloria y Pino-Juste, 2020). No obstante, respecto al nivel de especialización en Música del profesorado español se podría considerar que disponen de una capacitación mayor que la de los países analizados -si elimináramos la variable de la duración de la formación inicial-. En este sentido, para Educación Primaria, el profesorado español se considera semiespecialista como también podría serlo el alemán de NRW (puesto que es especialista en dos materias), a diferencia del austríaco que es generalista. Del mismo modo, para Educación Secundaria, mientras que el profesorado de Austria y Alemania (NRW) es especialista en dos materias, en el contexto español son especialistas exclusivamente en Música.

A partir de los resultados obtenidos se puede observar cómo la educación musical en España, pese a ser optativa, mantiene una presencia aceptable respecto a la de países con una larga tradición musical como Alemania (NRW) y Austria. Asimismo, sus aprendizajes tampoco difieren mucho del resto de territorios analizados. En consecuencia, cabrá analizar otros factores que podrían ser más determinantes que el horario semanal de educación musical o los aprendizajes ofertados, como pueden ser la formación inicial docente (Aróstegui y Kyakuwa, 2021), la cultura musical de las familias o el contexto donde se desenvuelve el alumnado (McPherson et al., 2015). Respecto a los aprendizajes que se proyectan desde la Unión Europea sobre la educación musical cabrá seguir analizando otros países para valorar cómo está evolucionando esta gran disparidad entre países que apuntaban López-García y De Moya-Martínez (2017). Si bien, con el presente estudio podemos afirmar que no existe una gran diferencia de la enseñanza de la educación musical entre los tres territorios analizados.

\section{Referencias}

Arnove, R.F., Torres, C.A. y Franz, S. (2016). Educación comparada: la dialéctica de lo global y lo local. Tirant Humanidades. 
Aróstegui, J.L. (2016). Exploring the global decline of music education. Arts education policy review, 117(2), 96-103. https://doi.org/10.1080/10632913.2015.1007406

Aróstegui, J.L. (2020). Implications of neoliberalism and knowledge economy for music education. Music Education Research, 22(1), 42-53. https://doi.org/10.1080/14613808.2019.1703923

Aróstegui, J.L. y Kyakuwa, J. (2021). Generalist or specialist music teachers? Lessons from two continents. Arts Education Policy Review, 122(1), 19-31. https://doi.org/10.1080/10632913.2020.1746715

Bate, E. (2020). Justifying music in the national curriculum: The habit concept and the question of social justice and academic rigour. British Journal of Music Education, 37(1), 3-15. https://doi.org/10.1017/S0265051718000098

Belletich, O., Wilhelmi, M.R. y Ángel-Alvarado, R. (2016). La educación musical en la formación básica en España. El problema de la dispersión curricular. Perspectiva Educacional. Formación de Profesores, 55(2), 158-170. http://dx.doi.org/10.4151/07189729-Vol.55-Iss.2-Art.454

Brasche, I. y Thorn, B. (2018). Addressing dimensions of "The Great Moral Wrong": How inequity in music education is polarizing the academic potential of Australian students. Arts Education Policy Review, 119(3), 124-136. https://doi.org/10.1080/10632913.2016.1201029

Bundesgesetzblatt für die Republik Österreich (2017). 138. Bundesgesetz: Bildungsreformgesetz. NR:GPXXVIA2254/A $\quad$ AB 1707 S. $188 . \quad$ BR: $\quad$ AB $9852 \quad$ S. 871. https://www.ris.bka.gv.at/Dokumente/BgblAuth/BGBLA_2017_I_138/BGBLA

Bundesministerin für Bildung, Wissenschaft und Kultur (2004). Verordnung mit der die Verordnung über die Lehrpläne der allgemein bildenden höheren Schulen geändert wird. BGB1. II Nr. 277. https://www.bmbwf.gv.at/Themen/schule/schulrecht/gvo/vo

Bundesministerin für Unterricht, Kunst und Kultur (2012a). Verordnung mit welcher die Lehrpläne der Volksschule und der Sonderschulen erlassen warden. BGB1. Nr.134/1963 in der Fassung BGB1. II Nr. 303/2012. https://www.bmbwf.gv.at/Themen/schule

Bundesministerin für Unterricht, Kunst und Kultur (2012b). Verordnung der mit der die Lehrpläne der Neue Mittelschulen erlassen. BGBl. II Nr. 185/2012. https://www.bmbwf.gv.at/Themen/schule/schulrecht/gvo/nms_umsetzungspaket.html

@Borja Mateu Luján. The content of this article is the sole responsibility of the authors. The Revista Electrónica de LEEME and Universitat de València are not liable for any legal actions that may arise involving the article's content. Revista Electrónica de LEEME - Lista Electrónica Europea de Música en la Educación-. http://ojs.uv.es/index/php/LEEME/index ISSN: 1575-9563. Editores: Universidad de Valencia y Jesús Tejada. Visibilidad de esta revista: SCOPUS, Emerging Sources Citation Index (Clarivate), EBSCO, CINDOC (CSIC), Citefactor, COPAC, Dialnet, DICE (CSIC), DOAJ, e-revistas (CSIC), EBSCO Premier, ERIH+, Gale Cengage Learning, IN-RECS, IRESIE, LATINDEX, MIAR, OCLC Worldcat, RESH, REDIB, RILM Core Journals, SUDOC, ULRICHS. Esta revista es de acceso libre mediante licencia Creative Commons $4.0 \mathrm{CC}$ by. Política de archivado: etiqueta verde SHERPA-ROMEO. 
Campbell, P.S. (2020). At the nexus of ethnomusicology and music education: pathways to diversity, equity, and inclusion. Arts Education Policy Review, 121(3), 106-110. https://doi.org/10.1080/10632913.2019.1709936

Carmona Fernández, J.J. y Jurado Fernández, M. (2010). Formación del profesorado de música en Europa meridional: Chipre, Grecia, Malta y Portugal. Profesorado. Revista de Currículum y Formación del Profesorado, 14(2), 57-65. http://www.ugr.es/local/recfpro

Carrillo-Aguilera, C., Viladot-Vallverdú, L. y Pérez-Moreno, J. (2017). Impacto de la educación musical: una revisión de la literatura científica. Revista Electrónica Complutense de Investigación en Educación Musical, 14, 61-74. https://doi.org/10.5209/RECIEM.54828

Casanova-López, O. y Serrano-Pastor, R.M. (2018). La Educación Musical en el actual currículo español. ¿Qué formación recibe el alumnado en la enseñanza Primaria? Revista Electrónica Complutense de Investigación en Educación Musical, 15, 3-17. http://dx.doi.org/10.5209/RECIEM.54844

Chrystosomou, S. (2015). Evaluación en clase de música: retos inherentes y la problemática de PISA. Revista Internacional de Educación Musical, 3, 23-30. http://dx.doi.org/10.12967/RIEM-2015-3-p023-030

Comisión Europea (2012). Un nuevo concepto de educación: invertir en las competencias para lograr mejores resultados socioeconómicos (52012DC0669). https://eurlex.europa.eu

Domínguez-Lloria, S. y Pino-Juste, M. (2020). Análisis comparativo de la formación inicial del profesorado de música de primaria y secundaria en Europa. Revista Electrónica de LEEME, 46, 224-239. https://doi.org/10.7203/LEEME.46.18033

Escalante, S. (2020). Exploring access, intersectionality, and privilege in undergraduate music education courses. Journal of Music Teacher Education, 29(2), 22-37. https://doi.org/10.1177/1057083719873981

Esteve-Faubel, J.M. (2019). La educación musical y el comparatismo. Revista Española de Educación Comparada, 34, 41-61. https://doi.org/10.5944/reec.34.2019.24243

Eurydice (2020). The Structure of the European Education Systems 2020/21: Schematic Diagrams. Eurydice Facts and Figures. Publications Office of the European Union. http://dx.doi.org/10.2797/39049

Fernández-Jiménez, A. y Jorquera-Jaramillo, M.C. (2017). El sentido de la educación musical en una educación concebida como motor de la economía del conocimiento: una propuesta

@Borja Mateu Luján. The content of this article is the sole responsibility of the authors. The Revista Electrónica de LEEME and Universitat de València are not liable for any legal actions that may arise involving the article's content. Revista Electrónica de LEEME - Lista Electrónica Europea de Música en la Educación-. http://ojs.uv.es/index/php/LEEME/index ISSN: 1575-9563. Editores: Universidad de Valencia y Jesús Tejada. Visibilidad de esta revista: SCOPUS, Emerging Sources Citation Index (Clarivate), EBSCO, CINDOC (CSIC), Citefactor, COPAC, Dialnet, DICE (CSIC), DOAJ, e-revistas (CSIC), EBSCO Premier, ERIH+, Gale Cengage Learning, IN-RECS, IRESIE, LATINDEX, MIAR, OCLC Worldcat, RESH, REDIB, RILM Core Journals, SUDOC, ULRICHS. Esta revista es de acceso libre mediante licencia Creative Commons 4.0 CC by. Política de archivado: etiqueta verde SHERPA-ROMEO. 
de marco filosófico. Revista Electrónica Complutense de Investigación en Educación Musical, 14, 95-107. http://dx.doi.org/10.5209/RECIEM.54834

Garvis, S., Barton, G. y Hartwig, K. (2017). Music Education in Schools: what is taught? A comparison of curriculum in Sweden and Australia. Australian Journal of Music Education, 51(2), 10-19. http://eprints.usq.edu.au/id/eprint/34795

Herrera-Torres, L., Lorenzo Quiles, O. y Ocaña Fernández, A. (2010). Formación inicial del profesorado de música en Irlanda y Reino Unido. Profesorado, Revista de Currículum y Formación del Profesorado, 14(2), 111-126. http://www.ugr.es/local

Horsley, S. (2015). Facing the music: Pursuing social justice through music education in a neoliberal world. En C. Benedict, P. Schimdt, G. Spruce y P. Woodford (Eds.), The Oxford handbook of social justice in music education (pp.62-77). http://dx.doi.org/10.1093/oxfordhb/9780199356157.013.6

Iotova, A.I. y Siebenaler, D. (2018). La formación del profesorado de música en la Universidad Complutense de Madrid y la Universidad Estatal de California: Un estudio comparado. Profesorado, Revista de Currículum y Formación del Profesorado, 22(3), 295-315. https://doi.org/10.30827/profesorado.v22i3.8003

Jefatura del Estado (2013). Ley Orgánica 8/2013, 9 de diciembre, para la mejora de la calidad educativa. BOE (10/12/2013), núm.295, referencia 12886, pp.97858-97921. https://www.boe.es/eli/es/lo/2013/12/09/8

Lehmann-Wermser, A. (2015). Teaching Music in Germany. En S. Figueiredo, J. Soares y R.F. Schambeck (Eds.) The preparation of music teachers (pp.219-240). ANPPOM.

López-García, N.J. (2018). Educación musical y currículo en la enseñanza primaria española: de la legislación general a la concreción autonómica. Revista da Abem, 26(41), 56-76. https://doi.org/10.33054/ABEM2018b4104

López-García, N.J. y De Moya-Martínez, M.V. (2017). Documentos clave de la Unión Europea sobre educación musical en las enseñanzas obligatorias. Revista Electrónica Complutense de Investigación en Educación Musical, 14, 171-186. https://doi.org/10.5209/RECIEM.52409

Mateu-Luján, B. (2021a). Estudio comparado de la educación musical en los sistemas educativos europeos de Alemania, Reino Unido, Países Bajos, Austria y España. (Tesis doctoral). Universidad de Valencia. https://www.proquest.com/docview/2578056725/abstract

@Borja Mateu Luján. The content of this article is the sole responsibility of the authors. The Revista Electrónica de LEEME and Universitat de València are not liable for any legal actions that may arise involving the article's content. Revista Electrónica de LEEME - Lista Electrónica Europea de Música en la Educación-. http://ojs.uv.es/index/php/LEEME/index ISSN: 1575-9563. Editores: Universidad de Valencia y Jesús Tejada. Visibilidad de esta revista: SCOPUS, Emerging Sources Citation Index (Clarivate), EBSCO, CINDOC (CSIC), Citefactor, COPAC, Dialnet, DICE (CSIC), DOAJ, e-revistas (CSIC), EBSCO Premier, ERIH+, Gale Cengage Learning, IN-RECS, IRESIE, LATINDEX, MIAR, OCLC Worldcat, RESH, REDIB, RILM Core Journals, SUDOC, ULRICHS. Esta revista es de acceso libre mediante licencia Creative Commons 4.0 CC by. Política de archivado: etiqueta verde SHERPA-ROMEO. 
Mateu-Luján, B. (2021b). La Educación Musical en España dentro del currículo obligatorio de educación secundaria. Estudio comparado entre comunidades autónomas. Revista Española de Educación Comparada, 37, 338-354. https://doi.org/10.5944/reec.37.2021.27541

McPherson, G.E., Osborne, M.S., Barrett, M.S., Davidson, J.W. y Faulkner, R. (2015). Motivation to study music in Australian schools: The impact of music learning, gender, and socio-economic status. Research Studies in Music Education, 37(2), 141-160. https://doi.org/10.1177/1321103X15600914

Ministerio de Educación, Cultura y Deporte (2014). Real Decreto 126/2014, de 28 de febrero, por el que se establece el currículo básico de la Educación Primaria. BOE (01/03/2015), núm.52, referencia 2222, pp.19349-19420. http://www.boe.es/boe/dias/2014/03/01/

Ministerio de Educación, Cultura y Deporte (2014). Real Decreto 1105/2014, de 26 de diciembre, por el que se establece el currículo básico de la Educación Secundaria Obligatoria y del Bachillerato. BOE (03/01/2015), núm.3, pp.169-546. https://www.boe.es/eli/es/rd

Ministerium für Schule und Bildung des Landes Nordrhein-Westfalen (2005). Verordnung über den Bildungsgang in der Grundschule (Ausbildungsordnung Grundschule - AO-GS) (1311 Nr. 1.1). Vom 23. März 2005 zuletzt geändert durch Verordnung vom 26. 1. Mai 2021 (SGV. NRW. 223). https://bass.schul-welt.de/pdf/6181.pdf?20210701075933

Ministerium für Schule und Bildung des Landes Nordrhein-Westfalen (2008). Richtlinien und Lehrpläne für die Grundschule. RdErl. Des Ministeriums für Schule und Weiterbildung v. 16.7.08 - 511 - 6.03.12.02 - 4419. https://www.schulentwicklung.nrw.de

Ministerium für Schule und Bildung des Landes Nordrhein-Westfalen (2011). Sekundarstufe IGymnasium; Richtlinien und Lehrpläne; Kernlehrpläne für die Fächer Kunst, Musik, Evangelische Religionslehre, Katholische Religionslehre und Sport. RdErl. $d$. Ministeriums für Schule und Weiterbildung v. 11.05.2011 - 532 -6.08.01.13 - 94565. https://www.schulentwicklung.nrw.de/lehrplaene/upload/

Ministerium für Schule und Bildung des Landes Nordrhein-Westfalen (2012a). Verwaltungsvorschriften zur Verordnung über die Ausbildung und die Abschlussprüfungen in der Sekundarstufe I (VVzAPO-S I). RdErl. d. Ministeriums für Schule und Weiterbildung v. 28.06.2019 (ABl. NRW. 08/19). Vom 2. November 2012 zuletzt geändert durch Verordnung vom 1. Mai 2021 (SGV. NRW. 223) https://bass.schul-welt.de/pdf/12691.pdf?20210701071735

Ministerium für Schule und Bildung des Landes Nordrhein-Westfalen (2012b). Sekundarstufe I - Gesamtschule; Richtlinien und Lehrpläne; Kernlehrpläne Kunst, Musik und Sport.

@Borja Mateu Luján. The content of this article is the sole responsibility of the authors. The Revista Electrónica de LEEME and Universitat de València are not liable for any legal actions that may arise involving the article's content. Revista Electrónica de LEEME - Lista Electrónica Europea de Música en la Educación-. http://ojs.uv.es/index/php/LEEME/index ISSN: 1575-9563. Editores: Universidad de Valencia y Jesús Tejada. Visibilidad de esta revista: SCOPUS, Emerging Sources Citation Index (Clarivate), EBSCO, CINDOC (CSIC), Citefactor, COPAC, Dialnet, DICE (CSIC), DOAJ, e-revistas (CSIC), EBSCO Premier, ERIH+, Gale Cengage Learning, IN-RECS, IRESIE, LATINDEX, MIAR, OCLC Worldcat, RESH, REDIB, RILM Core Journals, SUDOC, ULRICHS. Esta revista es de acceso libre mediante licencia Creative Commons 4.0 CC by. Política de archivado: etiqueta verde SHERPA-ROMEO. 
RdErl. d. Ministeriums für Schule und Weiterbildung v. 10.10.2012 - 532 - 6.03.15.06105207. https://www.schulentwicklung.nrw.de/lehrplaene/upload/klp_SI/GE/musik/

Ministerium für Schule und Bildung des Landes Nordrhein-Westfalen (2012c). Sekundarstufe IHauptschule; Richtlinien und Lehrpläne; Kernlehrpläne Arbeitslehre, Kunst, Musik, Sport und Textilgestaltung. RdErl. d. Ministeriums für Schule und Weiterbildung v. 12.12.2012 - 532 - 6.03.15.06-105207. https://www.schulentwicklung.nrw.de

Ministerium für Schule und Bildung des Landes Nordrhein-Westfalen (2012d). Sekundarstufe I - Realschule; Richtlinien und Lehrpläne; Kernlehrpläne Kunst, Musik, Sport und Textilgestaltung RdErl. d. Ministeriums für Schule und Weiterbildung v. 13.11 .2012 532 - 6.03.15.06-105207. https://www.schulentwicklung.nrw.de

Ministerium für Schule und Bildung des Landes Nordrhein-Westfalen (2019). Sekundarstufe lGymnasium; Richtlinien und Lehrpläne; 17 Kernlehrpläne für die Pflichtfächer. RdErl. d. Ministeriums für Schule und Bildung v. 23.06.2019 - 526-6.03.13.02-143664. https://www.schulentwicklung.nrw.de/lehrplaene/lehrplan/207

Morales, A. (2017). Presentación: La educación musical, una mirada del pasado al futuro. Revista Española de Pedagogía, 75(268), 389-398. https://www.jstor.org/stable/26379284

Palmer, E.S. (2018). Literature Review of Social Justice in Music Education: Acknowledging Oppression and Privilege. Update: Applications of Research in Music Education, 36(2), 22-31. https://doi.org/10.1177/8755123317711091

Phillips, D. y Schweisfurth, M. (2014). Comparative and international education: An introduction to theory, method, and practice. A\&C Black.

Rodríguez-Quiles, J. A. (2010). Formación inicial del profesorado de música en Alemania y Austria: Una perspectiva centroeuropea. Profesorado. Revista de Currículum y Formación del Profesorado, 14(2), 13-28. http://www.ugr.es/local/

Ruiz, G. y García-Álvarez, A. (2016). Music education at hospital schools in Spain and Sweden: Paths between governing and knowledge. European Education, 48(4), 258-273. https://doi.org/10.1080/10564934.2016.1239297

Salvador, K. y Kelly-McHale, J. (2017). Music teacher educator perspectives on social justice. Journal of Research in Music Education, 65(1), 6-24. https://doi.org/10.1177\%2F0022429417690340 


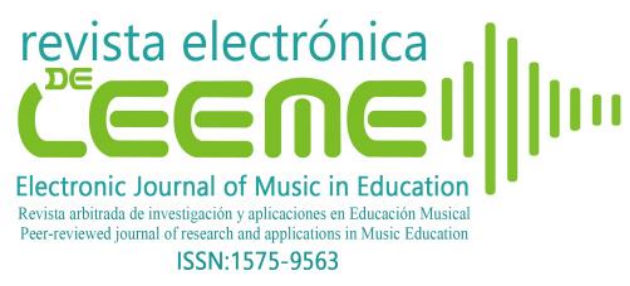

UNESCO (2015). Declaración de Incheon. Educación 2030: hacia una educación inclusiva y equitativa de calidad y un aprendizaje a lo largo de la vida para todos. https://unesdoc.UNESCO.org/ark:/48223/pf0000233813

Vicente-Nicolás, G. y Kirihara, A. (2012). Educación musical en Japón y en España: análisis del currículum para Educación Primaria. Profesorado: Revista de Currículum y Formación del Profesorado, 16(1), 379-390. http://www.ugr.es/local/recfpro/rev161ART1.pdf 